\title{
Application of Superhydrophobic Edge Effects in Solving the Liquid Outflow Phenomena
}

\author{
Jihua Zhang ${ }^{\dagger, \ddagger}$ Xuefeng Gao ${ }^{\dagger, *}$ and Lei Jiang ${ }^{\dagger, *}$ \\ Institute of Chemistry, Chinese Academy of Sciences, Beijing 100080, P. R. China. \\ Graduate School of the Chinese Academy of Sciences, Beijing 100049, P. R. China.
}

E-mail: jianglei@iccas.ac.cn; gaoxf@iccas.ac.cn

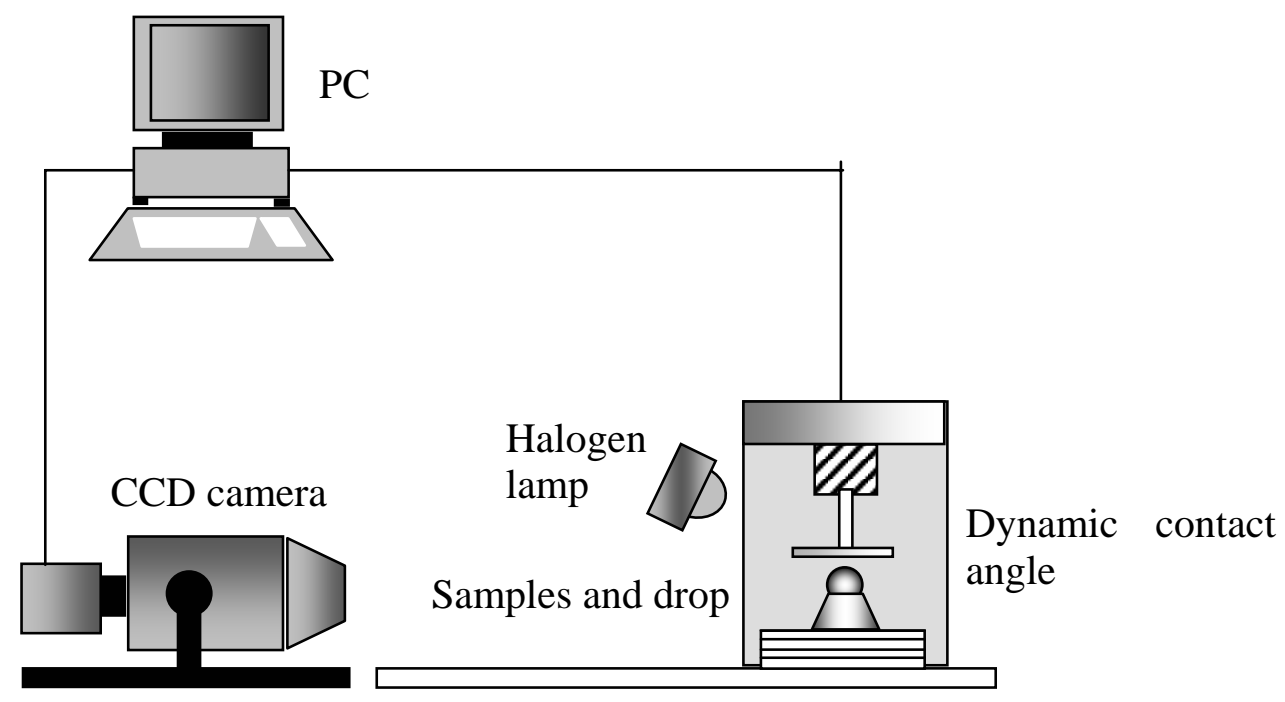

Figure S1 Schematic diagram of the experimental setup. 


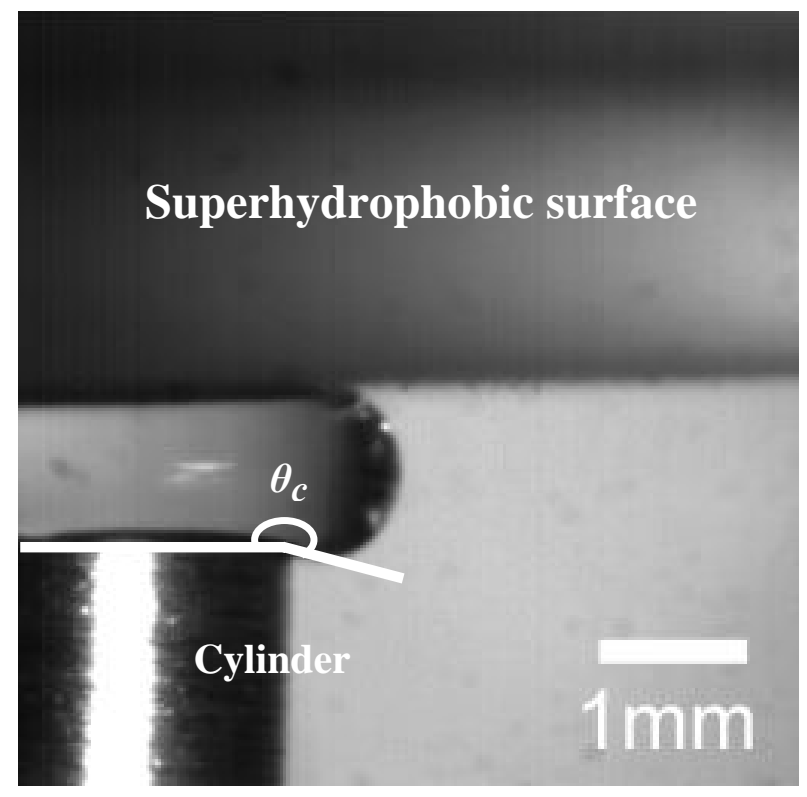

Figure S2 A drop pressed over the edge of FAS-Al samples by a superhydrophobic surface $\left(\omega=90^{\circ}\right.$, $\psi=9.8^{\circ}$ and $\left.d_{0}=4 \mathrm{~mm}\right)$. The contact angle $\theta_{c}$ was beyond $180^{\circ}$ relative to the top of cylinder. 


\begin{tabular}{|c|c|c|c|c|c|c|c|}
\hline Materials & $\theta_{a, 0} /^{\circ}$ & $d_{0} / \mathrm{mm}$ & $\omega /{ }^{\circ}$ & $\psi /^{\circ}$ & $\phi 1^{\circ}$ & $\theta_{c, \exp } /^{\circ}$ & $\theta_{c, \text { theo }}{ }^{\circ}$ \\
\hline \multirow{16}{*}{$\mathrm{Al}$} & \multirow{16}{*}{$83.6 \pm 2.1$} & \multirow{4}{*}{2.00} & 30 & 9.8 & 39.8 & 125.4 & 123.4 \\
\hline & & & 45 & 16.2 & 61.2 & 140.9 & 144.8 \\
\hline & & & 60 & 11.3 & 71.3 & 151.5 & 154.9 \\
\hline & & & 90 & 10.2 & 100.2 & 178.3 & 183.8 \\
\hline & & \multirow{4}{*}{3.00} & 30 & 12.3 & 42.3 & 125.9 & 125.9 \\
\hline & & & 45 & 13.2 & 58.2 & 145.7 & 141.8 \\
\hline & & & 60 & 6.5 & 66.5 & 142.3 & 150.1 \\
\hline & & & 90 & 11.8 & 101.8 & 176.3 & 185.4 \\
\hline & & \multirow{4}{*}{4.00} & 30 & 7.2 & 37.2 & 117.5 & 120.8 \\
\hline & & & 45 & 15.7 & 60.7 & 138.2 & 144.3 \\
\hline & & & 60 & 23.8 & 83.8 & 162.4 & 167.4 \\
\hline & & & 90 & 10.5 & 100.5 & 173.8 & 184.1 \\
\hline & & \multirow{4}{*}{5.00} & 30 & 5.3 & 35.3 & 122.3 & 118.9 \\
\hline & & & 45 & 32.9 & 77.9 & 160.5 & 161.5 \\
\hline & & & 60 & 21.7 & 81.7 & 163.9 & 165.3 \\
\hline & & & 90 & 9.5 & 99.5 & 178.1 & 183.1 \\
\hline \multirow{16}{*}{ FAS-Al } & \multirow{16}{*}{$110 \pm 1.7$} & \multirow{4}{*}{2.00} & 30 & 7.7 & 37.7 & 132.5 & 147.7 \\
\hline & & & 45 & 12.7 & 57.7 & 160.3 & 167.7 \\
\hline & & & 60 & 13.1 & 73.1 & 177.7 & 183.1 \\
\hline & & & 90 & 10.4 & 100.4 & 202.3 & 210.4 \\
\hline & & \multirow{4}{*}{3.00} & 30 & 6.7 & 36.7 & 140.3 & 146.7 \\
\hline & & & 45 & 10.3 & 55.3 & 168.7 & 165.3 \\
\hline & & & 60 & 11.4 & 71.4 & 178.6 & 181.4 \\
\hline & & & 90 & 8.3 & 98.3 & 195.3 & 208.3 \\
\hline & & \multirow{4}{*}{4.00} & 30 & 11.7 & 41.7 & 148.7 & 151.7 \\
\hline & & & 45 & 15.2 & 60.2 & 166.7 & 170.2 \\
\hline & & & 60 & 17.1 & 77.1 & 177.8 & 187.1 \\
\hline & & & 90 & 9.8 & 99.8 & 199.7 & 209.8 \\
\hline & & \multirow{4}{*}{5.00} & 30 & 10.1 & 40.1 & 150.1 & 150.1 \\
\hline & & & 45 & 14.1 & 59.1 & 166.3 & 169.1 \\
\hline & & & 60 & 17.3 & 77.3 & 176.7 & 187.3 \\
\hline & & & 90 & 10.1 & 100.1 & 203.1 & 210.1 \\
\hline
\end{tabular}

Table 1 The side features parameters of solid conical frusta and the critical advanced angles $\theta_{c}$. The angle $\theta_{c, \text { exp }}$ are the experimental critical values. The angle $\theta_{c, \text { theo }}$ are the calculated ones. 